\title{
SOME OBSERVATIONS ON EROSION MECHANISMS OF EB PVD TBCS
}

\author{
R.G Wellman and J.R Nicholls \\ School of Industrial and Manufacturing Science \\ Cranfield University \\ Bedford, MK 43 OAL
}

UK

\begin{abstract}
Following the successful application of Electron Beam (EB) Physical Vapour Deposition (PVD) Thermal Barrier Coatings (TBCs) to moving parts of turbine engines the erosion resistance of these coatings has been of interest among researchers. However, although there are a number of papers on the erosion rate of these coatings, little has been reported on their erosion mechanism. This paper provides observations on the erosion damage of EB PVD TBCs and discusses the type of damage caused by erosion as well as proposing a possible mechanism of erosion. The aim of the project as a whole was to model the erosion of EB PVD TBCs, but before modelling could begin it was necessary to determine the erosion mechanism of these coatings.
\end{abstract}

It was found that in all cases examined the erosion of the coatings proceeds through the accumulation of damage in the form of horizontal cracks in the columns of the coating and subsequent removal of the fractured sections. Since it appears as though the contact radius is important in the erosion process, the affect of varying the elastic properties of the erodent and the target on the contact radius was assessed.

Keywords: Erosion mechanisms, Thermal Barrier Coatings

\section{Introduction}

Since the advent of thermal barrier coatings (TBCs) more than three decades ago they have received a lot of attention with their promise of higher operating temperatures and more efficient turbine engines. However, most of 
the research carried out in the field of TBCs has concentrated on the thermal cyclic life of the coatings [1-4], the processing of the coatings [5-7] or the thermal conductivity of the coatings [8,9]. This is quite understandable since spalling due to oxide growth at the bond coat TBC interface and thermal stresses associated with engine operating cycles were the primary cause of failure in the early days of TBCs. However, with the introduction of electron beam (EB) physical vapour deposition (PVD) TBCs and their application to moving components in the hot gas stream erosion has become a prime concern. EB PVD TBCs, due to their unique columnar microstructure are far more strain tolerant than their plasma sprayed (PS) counter parts and can thus be used under more exacting operating conditions. It is under these operating conditions that erosion of the coated components is of primary importance. With blade tip velocities approaching mach 1 the impact of small-ingested particles can cause significant damage to the coatings. Thus more recently some researchers have studied the erosion of both PS and EB PVD TBCs [10-12].

These papers generally report the erosion rates of various TBCs under a range of erosion conditions in some cases giving results as normalised erosion rates. But generally there is very little information on the actual erosion mechanism except in recent papers by Nicholls et al [11,13].

The primary aim of this study was to formulate a computer model that would predict the erosion rate of EB PVD TBCs in order to provide design engineers with a usable tool for predicting component life. But before a computer program can be written the erosion mechanism of the system to be modelled must be fully understood. Thus, the first stage of this project has been involved in determining the erosion mechanism of EB PVD TBCs, the findings of which are outlined in this paper.

It is perhaps necessary at this stage to mention the difference between foreign object damage (FOD) and erosion. Although FOD is strictly speaking still erosion, more and more it is falling under its own classification, the mechanisms of material removal are vastly different and as such need to be differentiated. Microstructurally, the difference between the two are really quite straight forward; in FOD there is gross deformation of the TBC with the formation of a crater and the bending and cracking of a number of columns on a single impact [13]. Whereas with erosion there is much less large-scale mechanical damage and plastic deformation is confined to the top of the 
columnar microstructure. Cracking of the TBC and an accumulation of damage are necessary before material is removed and there is no gross deformation of the coating.

Unfortunately due to the unique columnar microstructure of the EB PVD TBCs the classical brittle erosion mechanisms proposed by Lawn et al and Tilly [14-17], with which all are familiar, can not be used in modelling the erosion of EB PVD TBCs. This meant that a thorough investigation into the erosion mechanism of the coatings was necessary before it would be possible to write a computer program to predict material wastage.

\section{Erosion of TBCs}

Of the papers on the erosion of TBCs only a few comment on the actual erosion mechanism as opposed to merely reporting the measured erosion rates under various conditions. Previous studies of brittle erosion have shown that material removal occurs via the formation, propagation and intersection of cracks with other crack and the surface. However, in EB PVD TBCs due to the columnar microstructure the cracks formed during erosion neither intersects with each other or indeed the surface. Which, as stated earlier, means that established erosion mechanisms can not be used to model the erosion of EB PVD TBCs. The erosion of these coatings appears to be via the formation of cracks in the columns and subsequent removal of the section of the material above the cracks. It has been noted in the literature that on particle impact the cracks propagate through the columns perpendicular to the direction of column growth and that under most conditions the coatings exhibit a brittle type of response to erosion.

However, Nicholls et al [11] noted that at high temperatures, temperatures above the softening point of the erodent, the coatings exhibited a ductile response to erosion. Maximum erosion rate was observed at $30^{\circ}$ impact with the rates at $90^{\circ}$ less than that at room temperature. This pseudo-ductile response was attributed to the softening of the erodent, silica, which appears to facilitate the removal of fractured section at low impact angles. This trend was not observed when eroding with alumina. 


\section{The Microstructure of EB PVD TBCs}

The importance of EB PVD TBCs lies in their far greater ability to accommodate strain than their PS counterparts which is due to their columnar microstructure. Thus EB PVD TBCs are gaining increased prominence in strain critical applications over their PS counter parts even though the later is a more mature process and widely used.

When discussing the effect of microstructure on erosion rate for most engineering materials grain size is an important factor. This is especially significant for brittle materials and dual phase materials.

When dealing with TBCs one has to consider two very distinctive and very different microstructures, which can not be treated in the same way as conventional engineering materials or indeed in the same way as each other. The very nature of the microstructure of these two processes necessitates the definition of two new erosion mechanisms. Or at the very least major modifications to classical erosion mechanisms. The following two micrographs indicate the difference in microstructure of EB PVD and PS TBCs.

It is the columnar microstructure of the EB PVD coatings, which gives them their high strain tolerance due to the fact that under mechanical load or thermal cycling the ceramic columns can move thus accommodating the strain [18]. This option is not available to the PS coatings, although research effort is focused on microcracking the splat network to improve the degree of strain tolerance.

Since the microstructure of a material has a great influence on the erosion rate and mechanism one must have a good understanding of the microstructure before attempting to determine an erosion mechanism. Most authors adequately describe the microstructure of EB PVD TBCs as a tapered columnar structure due to the three dimensional growth which can result in inhibiting the growth of neighbouring columns.

The above description only adequately describes the structure of the coatings on a macroscopic level, but what of the structure of the columns themselves? Are they single grains or a number of grains, is there directionality in the 
column and what degree of porosity is there? These are all important factors which can affect both the erosion rate and the mechanism.

On examining back scattered SEM micrographs of the cross section of EB PVD TBCs it becomes apparent that there are three microstructural features of the columns in the EB PVD coatings which could be important. The most obvious and the easiest to see is the fern leaf dendritic type structure on the edge of the columns which appear to indicate a $45^{\circ}$ growth angle of the columns. These are probably related to the mobility of the atoms on condensation onto the column and appear to be similar to solidification dendrites, but are formed directly from the vapour phase. The second feature, which is not immediately apparent, is the concentric striations fanning horizontally across the individual columns. These are due to the rotation of the sample during the coating process, with the thickness of the bands being directly related to the rotation speed and local vapour deposition rate. The third feature, which is very difficult to see, is that the columns of the coating are made up of numerous smaller columnar crystallites, which are extremely closely packed. This feature is more easily visible when looking at the top surface of a coating.

Other factors which are likely to affect the erosion rate of EB PVD TBCs are the size of the columns and the orientation of the columns with respect to the substrate. However, these issues will not be discussed in this paper, but are to be examined further in a future paper.

\section{Experimental Test Sample Preparation}

In order to determine the erosion mechanism of the EB PVD TBCs eroded samples were sectioned, mounted in resin, polished and examined under the scanning electron microscope (SEM). The samples had all been eroded under various impact conditions in the Cranfield erosion rig a description of which can be found in a previous publication by Nicholls et al [13]. The samples were all $\mathrm{ZrO}_{2}-8 \mathrm{wt} \% \mathrm{Y}_{2} \mathrm{O}_{3}$ EB PVD TBCs and were eroded at room temperature and $910^{\circ} \mathrm{C}$ using $60 \mu \mathrm{m}$ silica and $100 \mu \mathrm{m}$ alumina as the erodent at velocities between $140 \mathrm{~m} / \mathrm{s}$ and $300 \mathrm{~m} / \mathrm{s}$. the coatings were all $200-300 \mu \mathrm{m}$ thick. The results of the erosion tests can also be found in the literature [11]. 


\section{Results}

Examination of the eroded TBC samples showed that there was little visible plastic deformation and there were a number of cracked columns indicating a brittle type of erosion mechanism as was expected from what was observed in the literature. A number of samples were examined under the SEM and micrographs taken at various points in order to facilitate the measuring and counting of cracks in the columns.

Most of the cracks were perpendicular to the direction of column growth, which implies that tensile stresses were responsible for the initiation and propagation of the fracture rather than compressive stresses. It must be noted that these types of cracks were not observed in regions of the coating, which had not been eroded, and are thus a direct result of the erosion process. In nearly all cases the cracks propagated across the whole diameter of the column, furthermore no indications were found of cracks propagating into neighbouring columns.

The micrographs were used to measure the width of the column at the point of fracture, the distance of the fracture from the top surface and, where possible, the distance from the bottom surface of the coating. Analysis of fracture size and position in the coatings led to a number of pertinent observations being made:

- There appears to be no correlation between the column diameter and the depth at which it fractures.

- The cracks are all perpendicular to the direction of column growth.

- The cracks appear not to propagate into neighbouring columns.

- $80 \%$ of fractures occurred in the top $20 \mu \mathrm{m}$ of the coating.

- Most of the fractured columns have only one fracture.

- The number of adjacent columns with cracks never exceeds nine but in the majority of cases is five or less.

These observations allow a number of hypotheses to be proposed on the erosion mechanism of the EB PVD TBCs: 
- Fracture depth is independent of column diameter, as can be seen from graph 5.

- Cracking is caused by tensile rather than compressive stresses, evidenced by the lack of shear cracking in the eroded coatings.

- It is expected that by reducing column diameter the erosion resistance would increase, experimental work is currently underway to validate this hypothesis.

- Under the experimental conditions used material removal is likely to occur after five adjacent columns have fractured, it is expected that this would change under different erosion conditions.

- There is a degree of damage accumulation before material is removed.

From this information one can perhaps propose an erosion mechanism which would be similar to the Radial/Lateral model proposed by Lawn and Swain[16]. A fracture is produced in the column, a certain distance beneath the surface impact region, which then propagates parallel to the surface of the coating until it reaches the edge of the column in which it was initiated and can thus travel no further. Due to the complexity of the situation this problem is currently being analysed by the use of finite element modelling (FEM) and will be the subject of a paper in the near future. It is hoped that the FEM will enable the depth of cracks to be predicted with a fair degree of accuracy as this is directly related to the erosion rate of the coatings.

The following graph shows the distribution of crack depth in a sample that has been eroded at $90^{\circ}$ impact at room temperature with $100 \mu \mathrm{m}$ rounded silica particles. An analysis of the distribution shows that it fits a lognormal type. The cracks up to a depth of $30 \mu \mathrm{m}$ are all considered to be due to particle impact while the origin of the cracks at the greater depths are not fully understood and are believed to be caused by other factors.

Apart from analysing the depth of the cracks, the number of adjacent columns with cracks at some depth was also determined. It was found that in the majority of cases no more than 5 columns in a row would be cracked. 


\section{Effect of Elastic Properties on the Contact Radius}

Most of the equations proposed in the literature to predict erosion rate make use of the material properties of both the target and the erodent. Although these properties are available for the bulk materials they are not the same for the coatings and the bulk values can only be used as guide lines in determining the properties of the coatings. Further, FEM also requires the use of these properties. It was thus decided to determine how sensitive the size of the Hertzian contact radius was to certain material properties under Hertzian contact conditions.

The problem of relating the contact forces to the momentum of impacting forces for spherical projectiles has

$$
F \max =1.14\left[\frac{\left(16 / 9 \pi^{2}\right)\left(m^{3} V^{6} R\right)}{\left(k_{1}+k_{2}\right)^{2}}\right]^{\frac{1}{5}}
$$

already been solved [19]. The solution gives the maximum force generated during impact between a projectile and a planar surface as:

Where $\mathrm{V}, \mathrm{m}$ and $\mathrm{R}$ are the velocity, mass and radius of the projectile and $\mathrm{k}_{1}$ and $\mathrm{k}_{2}$ are the constants relating to the elastic properties of the target and projectile.

$$
\begin{aligned}
& k_{1}=\frac{\left(1-v_{1}^{2}\right)}{\pi E_{1}} \\
& k_{2}=\frac{\left(1-v_{2}^{2}\right)}{\pi E_{2}}
\end{aligned}
$$

Since this equation also applies when limited plastic deformation occurs at the point of impact it can be used for

$$
F \max =1.52 R^{2}\left(\frac{\pi V^{6} \rho^{3}}{\left(k_{1}+k_{2}\right)^{2}}\right)^{\frac{1}{5}}
$$


ceramic-ceramic impact problems. By inserting $m=4 / 3 \pi R^{3} \rho$ (where $\rho$ is the particle density) the equation can be reduced to:

This equation was substituted in to the Hertz equation and the contact radius was calculated for various values of the Poisson's ratio and Young's Modulus of the coating at various velocities and for different radii of the impacting particles at various velocities.

As can be seen from the above 3 graphs it appears as though velocity and the radius of the impacting particle have the greatest effect on the contact radius, which is to be expected. However, it also shows that relatively large variations in the Poisson's ratio and Young's modulus of the target has a negligible effect on the contact radius. This means that one can use the elastic properties of the bulk ceramic for modelling the erosion of the coatings. A similar effect was noted for variations in the Poisson's ratio and Young's modulus of the projectile.

\section{Erosion Mechanisms of EB PVD TBCs}

Due to the columnar morphology of TBCs, Hertzian ring cracks and cone cracks are highly unlikely to occur and the analysis of the samples shows no evidence of Hertzian type cracking.

After studying the micrographs of the cross sections of eroded TBCs it became evident that there were a number of different fracture mechanisms operating during the erosion of TBCs. The different mechanisms appear to operate in different regions of the coating and each of these shall be discussed separately.

\section{SHEAR CRACKING}

During all the analyses only one instance of shear cracking was observed and is mentioned here purely for the sake of completeness. The shear crack occurred on a sample that had been eroded at $910^{\circ} \mathrm{C}$ with alumina. From the micrograph one can see that a particle managed to form a wedge between two columns forcing them apart and initiating a shear crack. Since this was the only case of shear cracking in all the samples examined it was felt that 
this was not a mechanism that would affect the erosion rate to any great extent and would not be taken into account in the erosion model.

\section{NEAR SURFACE CRACKING}

Near surface cracking is defined as cracking that occurs within the first $20 \mu \mathrm{m}$ of the coating. This is the region, which accounts for the majority of the material removal during the erosion process. In this region there appears to be two mechanisms operating one of which is similar to the median lateral mechanism. As can be seen from the following micrograph the centre column shows evidence of classical median and lateral type cracking. One can assume that the impact centred on the middle of the column and it appears as though the median crack propagated along a sub grain boundary. This is consistent with classical median crack formation during point loading. However, it must be noted that the majority of fractured columns show no evidence of median cracks. So what is the mechanism of the other cracks in this region of the coating?

The question of inelastic deformation occurring in the TBC on impact is a difficult one to ascertain. Since the TBC has an average density of approximately $50 \%$ of the theoretical density, inelastic deformation is most likely to take place in the form of densification of the impacted column. It has not been possible yet to ascertain whether or not this occurs. As an increase in density would increase the local hardness, nanoindentation could possibly be used to determine whether densification occurs, a route that is currently being investigated.

It is proposed that the cracks in the columns are produced during the unloading part of the impact cycle, due to tensile stresses produced beneath the region of inelastic (plastic) deformation on unloading.

On initial loading of the impacted column a region of inelastic deformation is formed (possibly densification), this area is surrounded by a region of elastic deformation. On unloading of the sample the region of elastic deformation is prevented from relaxing as a result of the constraint provided by the region of inelastic deformation. This results in a tensile stress beneath the point of indentation in the direction of column growth and can initiate cracks parallel to the surface. 
It is assumed that the depth at which cracking will occur is directly related to the force of the impacting particle and the radius of the particle. Since these two factors will determine the size of the region of inelastic deformation they will also determine the depth at which cracking will occur.

\section{DENDRITE INITIATED CRACKING}

Dendrite initiated cracking typically occurs deeper that $20 \mu \mathrm{m}$ into the coating and is initiated from the 'base' of the dendritic column edge structure, rather than from the centre of the column. What causes these cracks and exactly how they are initiated has not yet been ascertained, but it is believed that the dendritic structure acts like a critical circumferential notch in a given column.

\section{MATERIAL REMOVAL}

Up until now the discussion has been about the different mechanisms by which cracking occurs in TBCs due to solid particle impact, now it is time to consider actual material removal. From the micrographs examined it is obvious that an accumulation of damage in the form of cracks is needed before material is removed. A single impact on an EB PVD TBCs will not necessarily result in material removal even though it might have fractured the column it impacted. This is attributed to the constraint of neighbouring columns 'holding' the fractured section in place. Only once a number of adjacent columns have been fractured can a subsequent impact result in material removal. When this does occur the fractured sections of a number of columns will be removed together.

From the analyses of both cross sectioned samples and the eroded surface of samples it seems reasonable to propose that material removal occurs when one column and all its first order neighbours have been cracked this would require removal from some seven columns simultaneously. This behaviour can be seen in figure 14 .

\section{Conclusions}

EB PVD TBCs crack under solid particle impact via a number of different mechanisms of which two appear to be predominant. These are:-

- Near Surface Cracking (Modified Median Lateral cracking) 
- Dendrite Initiated Cracking

The erosion of EB PVD TBCs proceeds via the accumulation of damage in a number of adjacent columns before material is lost.

\section{Future Work}

It is quite possible that the diameter of the columns could affect the erosion rate of the TBCs with a reduction in column diameter resulting in a reduction in wear rate. Since this will result in less material volume being removed per impact for smaller columns. A limit on this effect of column diameter is expected to exist. This limit will be determined by the column diameter at which one particle impact causes damage to more than one column.

There are four main sections of work, which need to be pursued for the continuation of this project:

1. The determination of the extent of plastic deformation occurring on impact of the TBC. This can possibly be achieved through the use of nanohardness tests.

2. The determination of crack depth on impact through the use of FEM.

3. Bringing all the information together in the form of a computer programme that can predict the erosion rate of EB PVD TBCs under various conditions.

4. Determine the effect of column diameter on the erosion rate of EB PVD TBCs.

\section{Acknowledgements}

The authors wish to thank EPSRC for their financial support of this work, under the RoPA award scheme. 
Thanks are also due to Mr Ken Lawson, Manager of Coating Services at Cranfield University, for the discussions on the vapour deposition process and its influence on the morphology of EB PVD coatings.

\section{Reference List}

1 T.A Cruse, S.E Stewart, and M Ortiz. Transactions of the ASME 110, 610-616 (1988).

2 B.C Wu, E Chang, S.F Chang, and C.H Chao. Thin Solid Films 172, 185-196 (1989).

3 J.H Sun, E Chang, B.C Wu, and C.H Tsai. Surface and Coatings Technology 58, 93-99 (1993).

4 D.M Nissley. Thermal Barrier Coatings Workshop NASA Conference Publication 3312 265-281 (1995).

5 Y Jaslier and S Alperine. AGARD Report 823, 8 (1998).

6 R.C Novak. NASA Conference Publication 3312 153-166 (1995).

7 W.A Kaysser, M Peters, K Fritscher, and U Schulz. AGARD Report 823, 9 (1998).

8 J.M Dorvaux. AGARD Report 823 (1998).

9 R.B Dinwiddie, S.C Beecher, B.A Nagaraj, and C.S Moore. NASA Conference Publication 3312167 (1995).

10 A.G Davis, D.H Boone, and A.V Levy. Wear 110, 101-116 (1986).

11 J.R Nicholls, Y Jaslier, and D.S Rickerby. Materials at High Temperature 15, 15-22 (1998).

12 T Rhys-Jones and F.C Toriz. High Temperature Technology 7, 73-81 (1989).

13 J.R Nicholls, Y Jaslier, and D.S Rickerby. 4th International Colloquia on Corrosion and Protection of Materials at High Temperatures, Les Embies France (1996).

14 B.R Lawn, A.G Evans, and D.B Marshall. J. of American Ceramic Society 574-581 (1980).

15 B.R Lawn and E.R Fuller. Journal of Materials Science 10 , 2016-2024 (1975). 
16 B.R Lawn and M.V Swain. Journal of Materials Science 10, 113-122 (1975).

17 G.P Tilly. Treatise on Materials Science \& Technology 13, 287-319 (1979).

18 P Morrell and D.S Rickerby. AGARD Report 823,20 (1998).

19 A.G Evans. Journal of the American Ceramic Society 56, 405-409 (1973). 


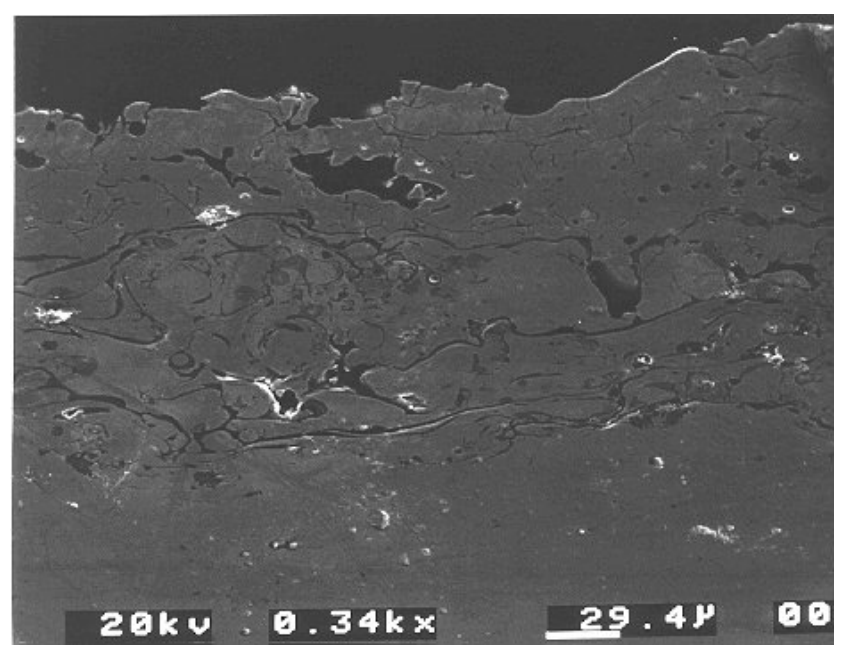

Figure 1: Micrograph of the microstructure of a PS TBC. 


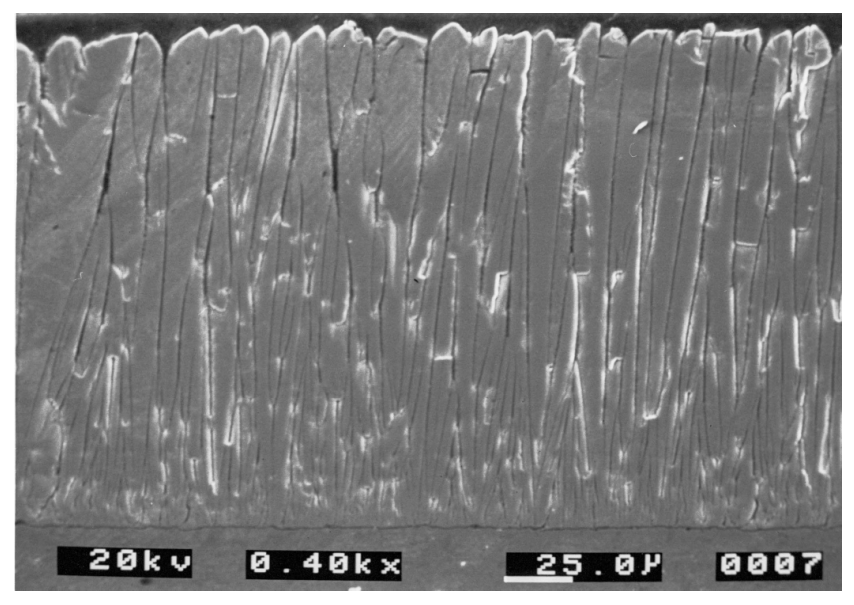

Figure 2: Micrograph of the microstructure of an EB PVD TBC. 


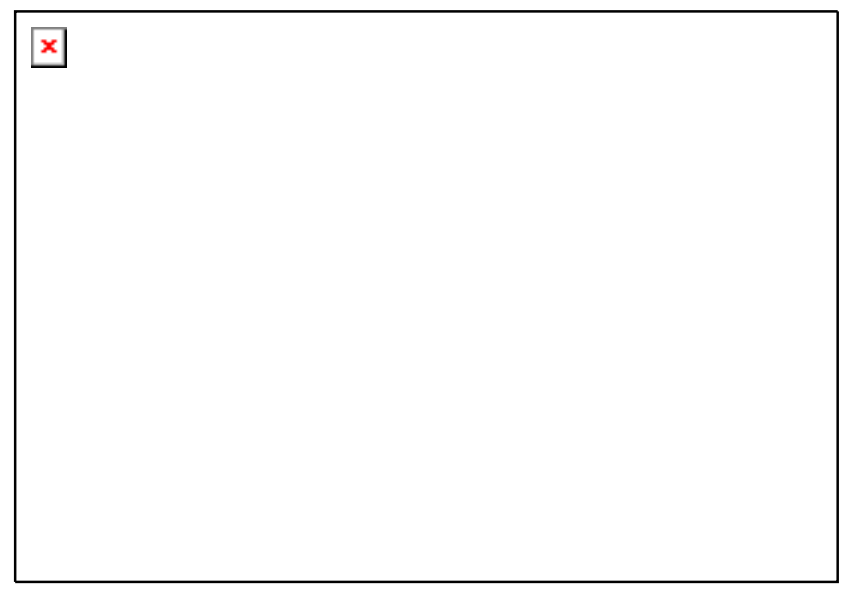

Figure 3: Fractograph of an EB PVD Coating showing the various microstructural features. 


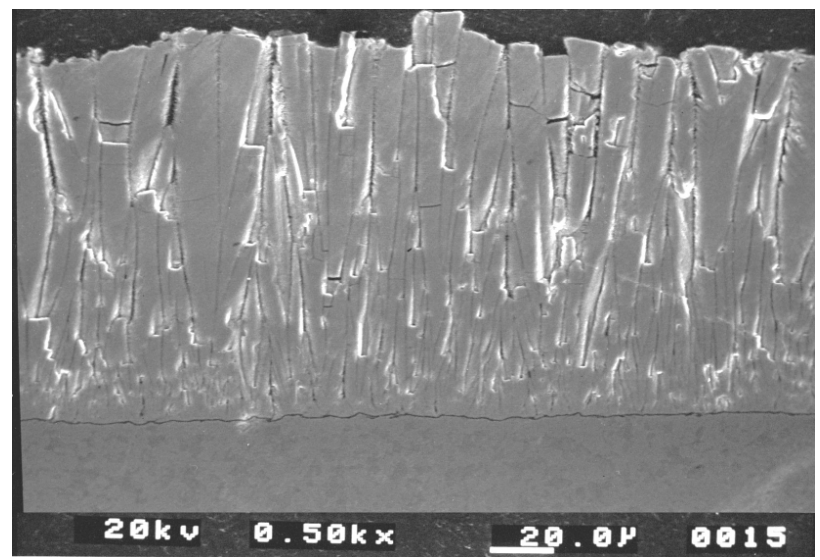

Figure 4: Micrograph showing cracking of the TBC columns due to erosion. 


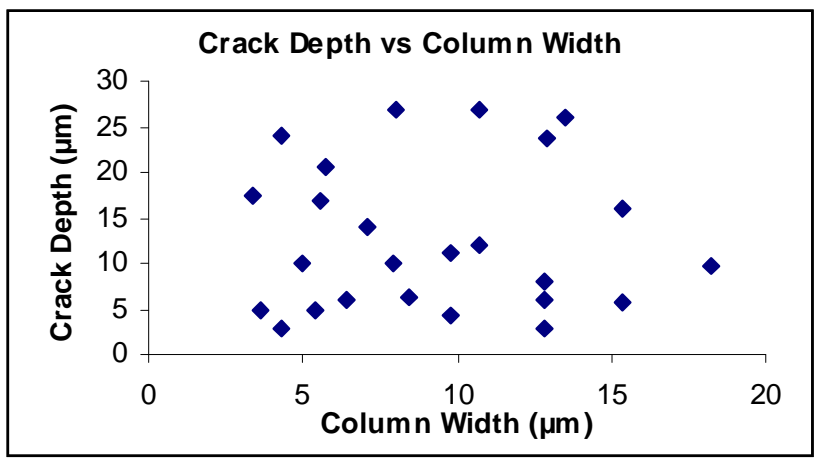

Figure 5: A plot of column width against crack depth showing that there is no correlation. 


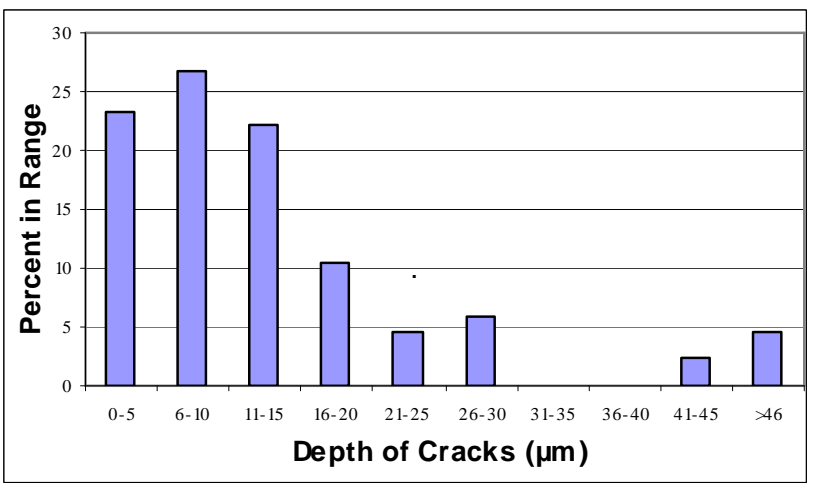

Figure 6: The distribution of crack depth in the TBCs examined. 


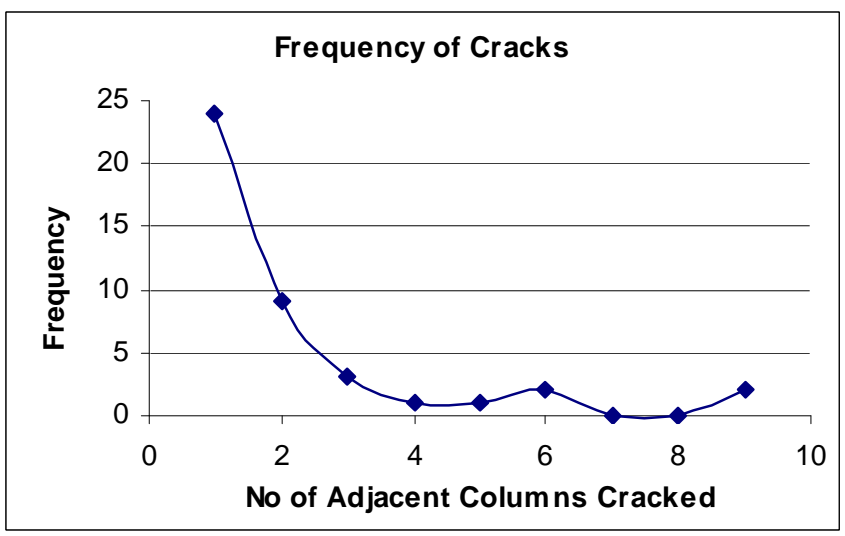

Figure 7: Plot of the number of adjacent columns with cracks vs frequency of occurrence. 


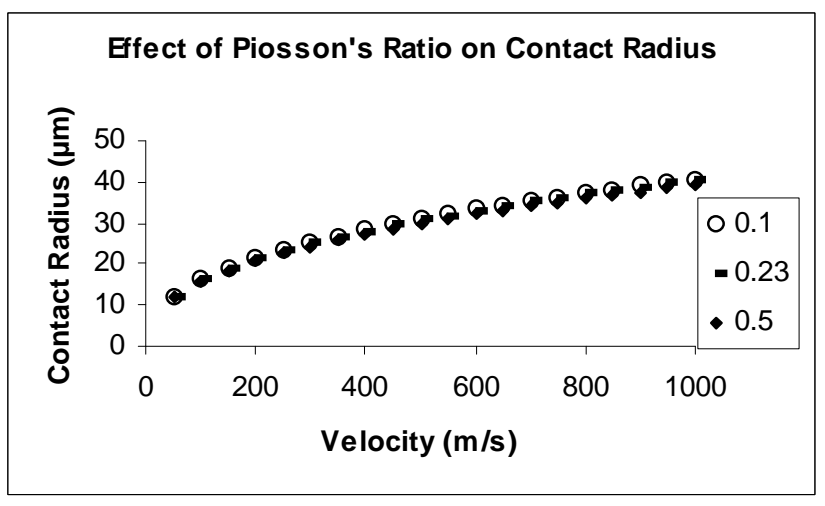

Figure 8: The effect of changing the target Poisson's ratio on the contact radius as a function of velocity. 


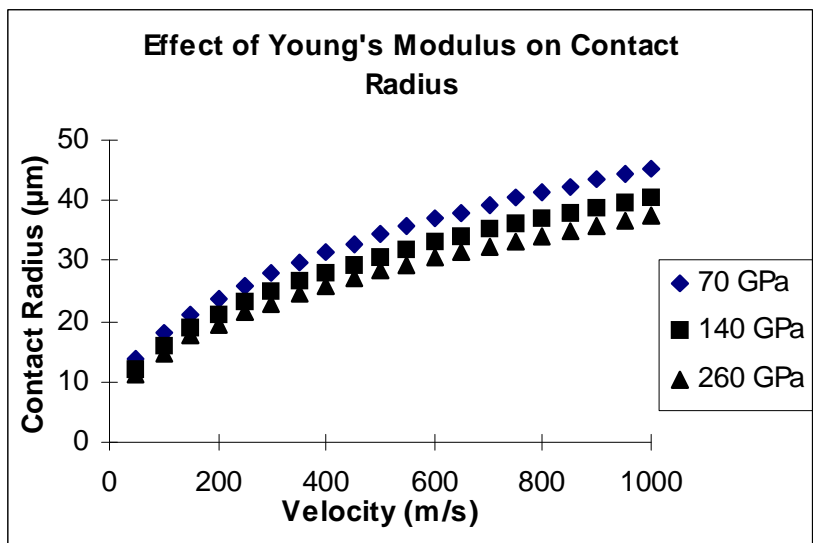

Figure 9: The effect of changing the target Young's Modulus on the contact radius under Hertzian contact conditions as a function of velocity. 


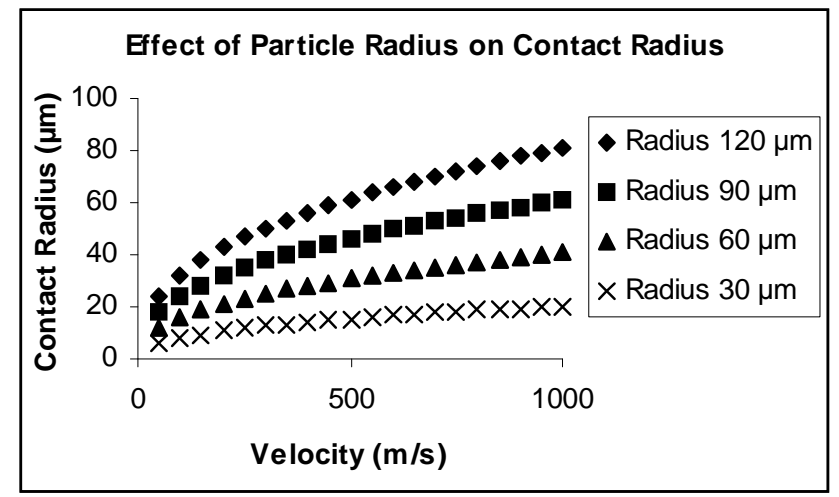

Figure 10: The effect of erodent particle radius on the contact radius under Hertzian contact conditions as a function of velocity. 


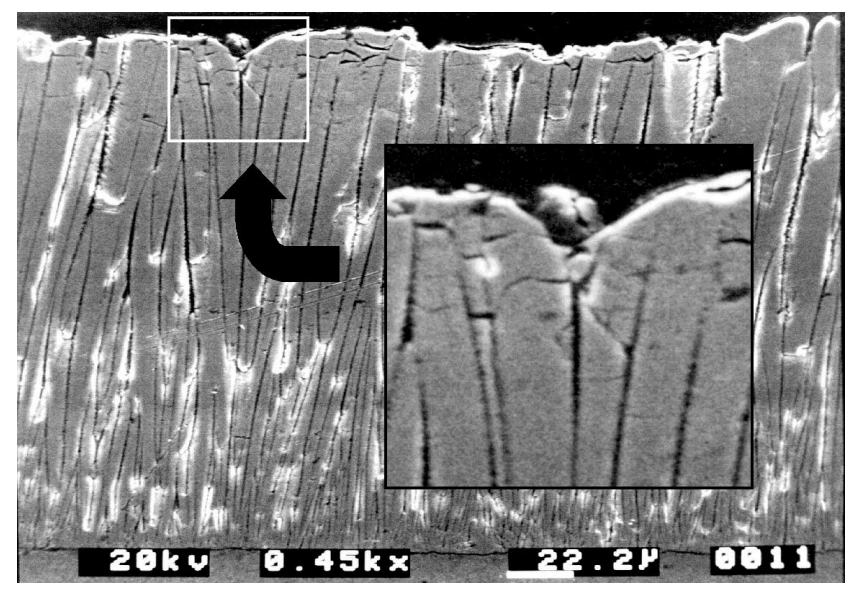

Figure 11: SEM micrograph showing cracking in a TBC note the single shear crack, enlarged in the insert. 


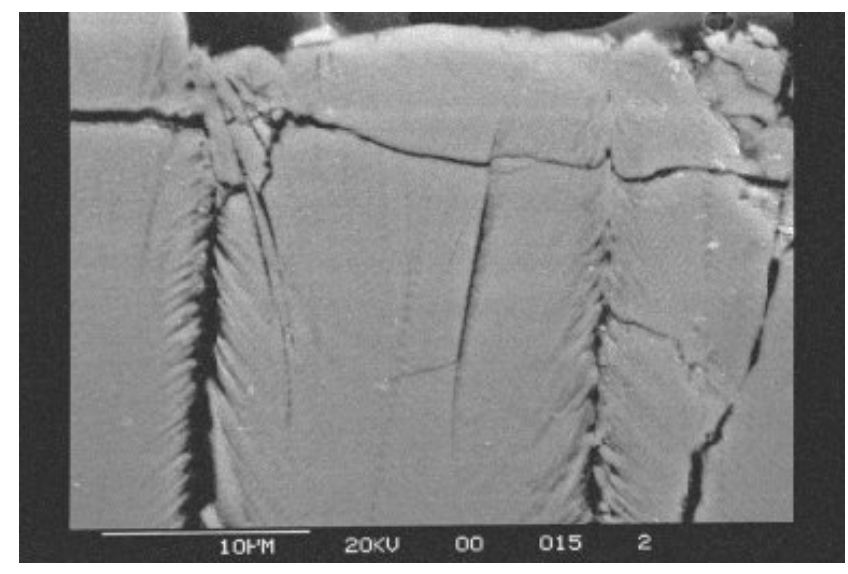

Figure 12: SEM micrograph showing median and lateral type cracking. 


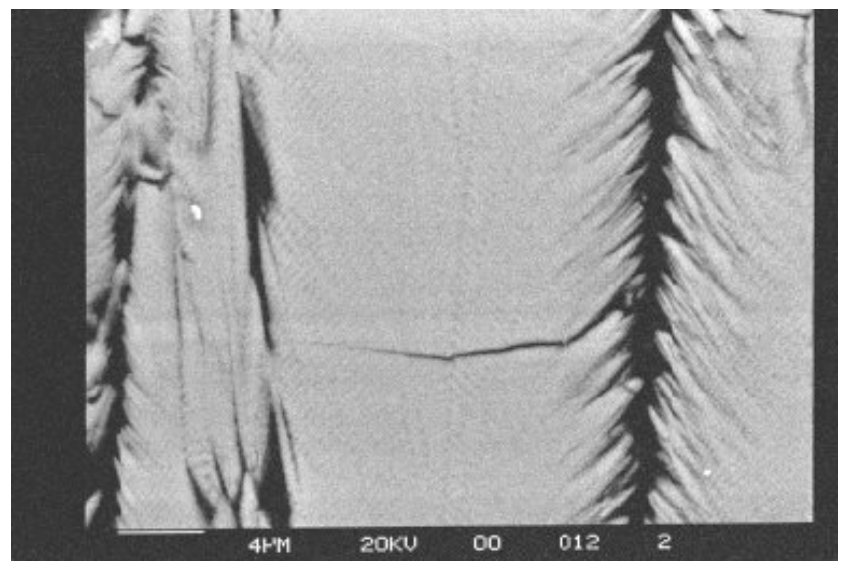

Figure 13: SEM micrograph showing a crack which has initiated from a dendrite. 


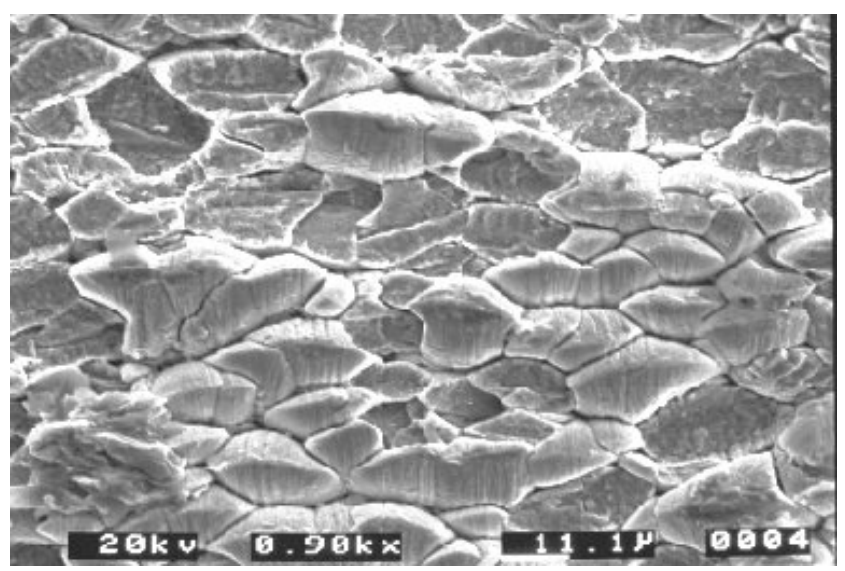

Figure 14: SEM micrograph of the surface of an eroded sample showing some regions where material has not been removed. 